SCIENTIFIC REPORT

\title{
Correlation of optical coherence tomography, with or without additional colour fundus photography, with stereo fundus fluorescein angiography in diagnosing choroidal neovascular membranes
}

\author{
S S Sandhu, S J Talks
}

Br J Ophthalmol 2005;89:967-970. doi: 10.1136/bjo.2004.060863

\begin{abstract}
Aims: To assess the diagnostic accuracy of optical coherence tomography (OCT), with/without colour fundus photographs, in predicting fundus fluorescein angiography (FFA) findings in patients suspected of havingchoroidal neovascularisation (CNV).

Methods: A consecutive series of patients suspected of havingCNV underwent OCT and stereo FFA images, which were assigned a diagnosis by two masked observers, one examining OCT alone and then OCT plus stereo colour photographs, and one examining FFA alone. The main outcome measures were the diagnostic accuracy of OCT (with/without colour photos) in predicting the presence of CNV and secondly the characteristics of the CNV compared to the FFA findings.

Results: 131 eyes of 118 patients were analysed. The sensitivity and specificity of OCT for detecting new potentially treatable CNV lesions compared to FFA was $96.4 \%$, and $66.0 \%$, respectively. For OCT with stereo images the sensitivity was $94.0 \%$ and specificity $89.4 \%$. For detecting CNV with a classic component the sensitivity and specificity of OCT alone was $78.6 \%$ and $82.7 \%$. With stereo colour images the sensitivity was $82.1 \%$ and specificity $89.3 \%$.

Conclusion: OCT is good at detecting the presence of CNV in patients suspected of having new CNV. However, it is less accurate at identifying the exact components of CNV. OCT cannot at present replace FFA in accurately diagnosing CNV components. However, this imaging method may have a role as a screening tool to help prioritise FFA requests.
\end{abstract}

$\mathrm{T}$ he advent of photodynamic therapy (PDT) has led to a large increase in fundus fluorescein angiography (FFA) requests to assess patients for choroidal neovascularisation (CNV). FFA is invasive, has a small but well recognised risk of severe complication, and is time consuming. As FFA is also required for treatment monitoring, the ophthalmic photography services have become heavily burdened. While information on lesion type and area is a prerequisite for PDT, and is best obtained by FFA, new treatments such as antivascular endothelial growth factor may only require knowledge of the diagnosis. OCT is non-contact, noninvasive, and quick to perform. The OCT findings in a wide variety of retinal pathologies, including $\mathrm{CNV}$, have been described..$^{1-6}$ With the advent of higher resolution, the OCT may have a role as a diagnostic tool for CNV. We, therefore, aimed to assess the accuracy of OCT, using FFA as the gold standard.

\section{MATERIALS AND METHODS}

A prospective series of images collected over a 6 month period from patients who had had OCT (Zeiss OCT3), stereo colour, and stereo FFA images (Zeiss FF450 IR) for a first time presentation of suspected CNV were examined. The images were taken as part of the department's routine assessment for such patients. The tests had been requested by a range of doctors with different levels of experience looking for a potentially treatable lesion, defined in our department, as a predominantly classic CNV with no pigment epithelial detachment (PED) (defined below). Images were reviewed by two independent observers, one assessing the OCT and then the OCT plus colour photographs (SSS), the other the FFA (SJT), each masked to the other's diagnostic classification and the clinical diagnosis. All OCT images were taken by an experienced operator, using the standard macular scanning protocol that involves six $6 \mathrm{~mm}$ radial scans. In addition, if any abnormality was detected, further cuts were taken through the lesion by moving the scan manually. The fast macular protocol was not used as this reduced the image quality required for lesion analysis.

The FFA images were classified into the following groups ${ }^{7-11}$ :

(1) Classic CNV, subdivided into $100 \%$ classic, classic without occult, predominantly classic with occult, or minimally classic with occult.

(2) Occult CNV subdivided as late leak of undetermined origin or vascularised PED. Retinal angiomatous proliferation (RAP) was also included.

(3) Serous PED-early area definition with increasing hyperfluorescence.

(4) Non-CNV—which could be defined as:

(a) Dry age related macular degeneration (AMD), drusen/ atrophy,

(b) Other diagnosis-for example, retinal vein occlusion (RVO), macular hole, etc.

The OCTs were classified as to whether there was an AMD lesion (defined by (1), (2), or (3) below), and further, to try to define the type of lesion with the aid of the following features: ${ }^{1212} 13$

(1) Classic CNV-a subretinal band corresponding to the retinal pigment epithelium (RPE) and choriocapillaris

Abbreviations: $A M D$, age related macular degeneration; CNV, choroidal neovascular membrane; FFA, fundus fluorescein angiography; OCT, optical coherence tomography; PDT, photodynamic therapy; PED, pigment epithelial detachment; RAP, retinal angiomatous proliferation; RPE, retinal pigment epithelium; RVO, retinal vein occlusion; SRF, subretinal fluid 


\begin{tabular}{|c|c|c|c|c|c|c|}
\hline & \multicolumn{3}{|c|}{ Classic component } & \multicolumn{3}{|c|}{ Occult } \\
\hline & $100 \%$ & Predominantly & Minimally & $\begin{array}{l}\text { Late } \\
\text { leak }\end{array}$ & $\begin{array}{l}\text { Vascularised } \\
\text { PED }\end{array}$ & RAP \\
\hline \multicolumn{7}{|l|}{ Classic CNV ( $\mathrm{n}=56$ ) } \\
\hline Classic with no occult ( $n=51)$ & 23 & 20 & 8 & & & \\
\hline $\begin{array}{l}\text { Predominantly classic with } \\
\text { occult }(n=2)\end{array}$ & & 2 & & & 2 & \\
\hline $\begin{array}{l}\text { Minimally classic with } \\
\text { occult }(n=3)\end{array}$ & & & 3 & 1 & 1 & 1 \\
\hline Occult CNV ( $n=25)$ & & & & 11 & 12 & 2 \\
\hline
\end{tabular}

which is thickened and disrupted, typically a fusiform or "cigar" shape with/without intraretinal/subretinal fluid (SRF).

(2) Occult CNV-a less well defined band than classic CNV but appears to be more sub-RPE with more disorganisation of the retina and subretinal and/or intraretinal fluid (cystoid).

(3) Serous PED-dome-shaped elevation of the reflective band corresponding to the RPE with an area of low reflectivity underneath.

(4) Non-CNV:

(a) Dry AMD, drusen/atrophy-thinning of the retinal layer with reduced reflectivity of the RPE but increased reflectivity of the choroidal layer

(b) Other diagnosis-for example, epiretinal membrane (ERM), macular hole.

Statistical analyses were calculated using Microsoft Excel and Graph Pad Instat version 3. As this study consisted of a review of tests performed as part of our routine clinical practice, ethics committee approval was not required.

\section{RESULTS}

FFA and OCT images of 128 patients were assessed. Ten patients were excluded because of the poor quality of the images. Thirteen patients had bilateral active pathology and so 131 eyes of 118 patients were analysed: mean age 73.2 years (SD 13.7), range 30-97); $57.6 \%$ female; $53.4 \%$ right eye. $\mathrm{CNV}$ associations were AMD in 126; myopic degeneration in three; punctate inner choroidopathy (PIC) in one; angioid streaks in one. The main diagnoses on FFA were 56 CNVs with a classic component (breakdown in table 1), 25 occult CNVs, three serous PEDs, 26 eyes with drusen/atrophy, and 21 other diagnoses: normal (six), RVO (three), cystoid macular oedema (CMO) (two), ERM (two), central serous retinopathy (two), diabetic retinopathy, laser scar with no recurrence, macroaneurysm, macular haemorrhage (no cause), malignant melanoma, and retinal pigment epithelium.

The sensitivity and specificity for detecting a potentially treatable lesion (classic containing CNV 56; occult CNV 25; serous PED 3) was for OCT alone $96.4 \%$ ( 81 of 84 ) and $66.0 \%$ (31 of 47), respectively, and for OCT with stereo images $94.0 \%$ ( 79 of 84 ) and $89.4 \%$ (42 of 47 ). In the group with no CNV or PED seen on FFA, 16 of $47(34.0 \%)$ were thought to have either CNV or PED on OCT, with the addition of stereo colour images this was reduced to five of 47 (10.6\%) (see tables 2 and 3 ).

The sensitivities, specificities and positive and negative predictive values for new AMD lesions and classic CNV are shown in table 4.

\section{DISCUSSION}

In 2002 we performed 321 FFAs looking for potentially treatable CNV. ${ }^{14}$ A fast, non-invasive diagnostic test such as OCT could help prioritise FFA requests.

In diagnosing new AMD we found that OCT alone had a sensitivity of $96.4 \%$ and a specificity of $66.0 \%$. OCT with stereo colour images had a sensitivity of $94.0 \%$ and a specificity of $89.4 \%$. The use of stereo colour images might be expected to provide similar information to clinical biomicroscopic examination, although may be not so good. As such, the use of OCT with clinical examination should give similar results. The typical FFA and OCT appearances of classic CNV, occult CNV, and serous PED and are shown in figure 1.

As with any diagnostic method, the number of patients with disease classed as without disease (false negative rate) is important. Only three were missed. On OCT, one patient was diagnosed with an ERM but on further review had evidence of SRF. Two patients were diagnosed as having only drusen/

\begin{tabular}{|c|c|c|c|c|c|c|}
\hline \multirow{2}{*}{\multicolumn{2}{|c|}{ FFA diagnosis }} & & \multicolumn{4}{|c|}{ OCT alone } \\
\hline & & & $\begin{array}{l}\text { Classic } \\
\text { CNV }\end{array}$ & $\begin{array}{l}\text { Occult } \\
\text { CNV }\end{array}$ & $\begin{array}{l}\text { Serous } \\
\text { PED }\end{array}$ & $\begin{array}{l}\text { No CNV (atrophy/ } \\
\text { drusen, other) }\end{array}$ \\
\hline \multicolumn{2}{|c|}{$\begin{array}{l}\text { Classic CNV } \\
\text { Non-classic CNV } \\
\text { Occult CNV }\end{array}$} & 56 & 44 & 9 & 0 & 3 \\
\hline & Late leak & 11 & 4 & 2 & 5 & 0 \\
\hline & Vasc PED & 12 & 0 & 2 & 10 & 0 \\
\hline & RAP & 2 & 1 & 1 & 0 & 0 \\
\hline \multirow{4}{*}{ No CNV } & Serous PED & 3 & 0 & 1 & 2 & 0 \\
\hline & & & & & & \\
\hline & Atrophy/drusen & 26 & 4 & 3 & 0 & 19 \\
\hline \multirow{2}{*}{\multicolumn{2}{|c|}{ Other diagnoses }} & 21 & 4 & 0 & 5 & 12 \\
\hline & & 131 & 57 & 18 & 22 & 34 \\
\hline
\end{tabular}




\begin{tabular}{|c|c|c|c|c|c|c|}
\hline \multirow[b]{2}{*}{ FFA diagnosis } & & & \multicolumn{4}{|c|}{ OCT with stereo images } \\
\hline & & & $\begin{array}{l}\text { Classic } \\
\text { CNV }\end{array}$ & $\begin{array}{l}\text { Occult } \\
\text { CNV }\end{array}$ & Serous PED & $\begin{array}{l}\text { No CNV (atrophy/ } \\
\text { drusen, other) }\end{array}$ \\
\hline \multirow[t]{5}{*}{$\begin{array}{l}\text { Classic CNV } \\
\text { Non-classic CNV } \\
\text { Occult CNV }\end{array}$} & & 56 & 46 & 6 & 1 & 3 \\
\hline & Late leak & 11 & 4 & 2 & 5 & 0 \\
\hline & Vasc PED & 12 & 0 & 1 & 11 & 0 \\
\hline & RAP & 2 & 1 & 0 & 0 & 1 \\
\hline & Serous PED & 3 & 0 & 0 & 2 & 1 \\
\hline \multicolumn{7}{|l|}{ No CNV } \\
\hline & $\begin{array}{l}\text { Atrophy/drusen } \\
\text { Other diagnoses }\end{array}$ & $\begin{array}{l}26 \\
21\end{array}$ & 2 & $\begin{array}{l}0 \\
1\end{array}$ & $\begin{array}{l}0 \\
1\end{array}$ & $\begin{array}{l}24 \\
18\end{array}$ \\
\hline Totals & & 131 & 54 & 10 & 20 & 47 \\
\hline
\end{tabular}

Table 4 Statistical analysis

\begin{tabular}{|c|c|c|c|c|}
\hline & $\begin{array}{l}\text { Sensitivity } \\
(95 \% \mathrm{Cl})\end{array}$ & $\begin{array}{l}\text { Specificity } \\
(95 \% \mathrm{Cl})\end{array}$ & $\begin{array}{l}\text { Positive predictive value } \\
(95 \% \mathrm{Cl})\end{array}$ & $\begin{array}{l}\text { Negative predictive value } \\
(95 \% \mathrm{CI})\end{array}$ \\
\hline \multicolumn{5}{|l|}{ OCT alone } \\
\hline New AMD lesion & $96.4 \%$ (90.0 to 99.3$)$ & $66.0 \%(50.6$ to 79.1$)$ & $83.5 \%(74.6$ to 90.3$)$ & $91.2 \%(76.3$ to 98.1$)$ \\
\hline Classic CNV & $78.6 \%(65.6$ to 88.4$)$ & $82.7 \%(72.2$ to 90.4$)$ & $77.2 \%(64.2$ to 87.3$)$ & $83.8 \%$ (73.4 to 91.3$)$ \\
\hline \multicolumn{5}{|l|}{ OCT with stereo images } \\
\hline New AMD lesion & $94.0 \%(86.7$ to 98.0$)$ & $89.4 \%$ (76.9 to 96.5$)$ & $94.1 \%(86.7$ to 98.0$)$ & $89.4 \%$ (76.9 to 96.5$)$ \\
\hline Classic CNV & $82.1 \%(69.6$ to 91.1$)$ & $89.3 \%(80.1$ to 95.3$)$ & $85.2 \%$ (72.9 to 93.4$)$ & $87.0 \%(77.4$ to 93.6$)$ \\
\hline
\end{tabular}

atrophy. Both had small CNVs, which we believe were missed by the cut of the OCT scan. One patient had a diagnosis of PIC and the other, AMD.

The false positive rate in diagnosing a new AMD lesion was $34.0 \%$ (16 of 47 ) by OCT alone. This was reduced to $10.6 \%$ (five of 47 ) with the addition of stereo colour images. On retrospective review of these OCT images, two were reclassified as drusen/atrophy and therefore as non-CNV-both FFA diagnoses were drusen/atrophy. The remaining 14 still had abnormal features on the OCT image. If the OCT had been used to "screen" for a potential CNV, these would have still warranted further investigation. Among these were two

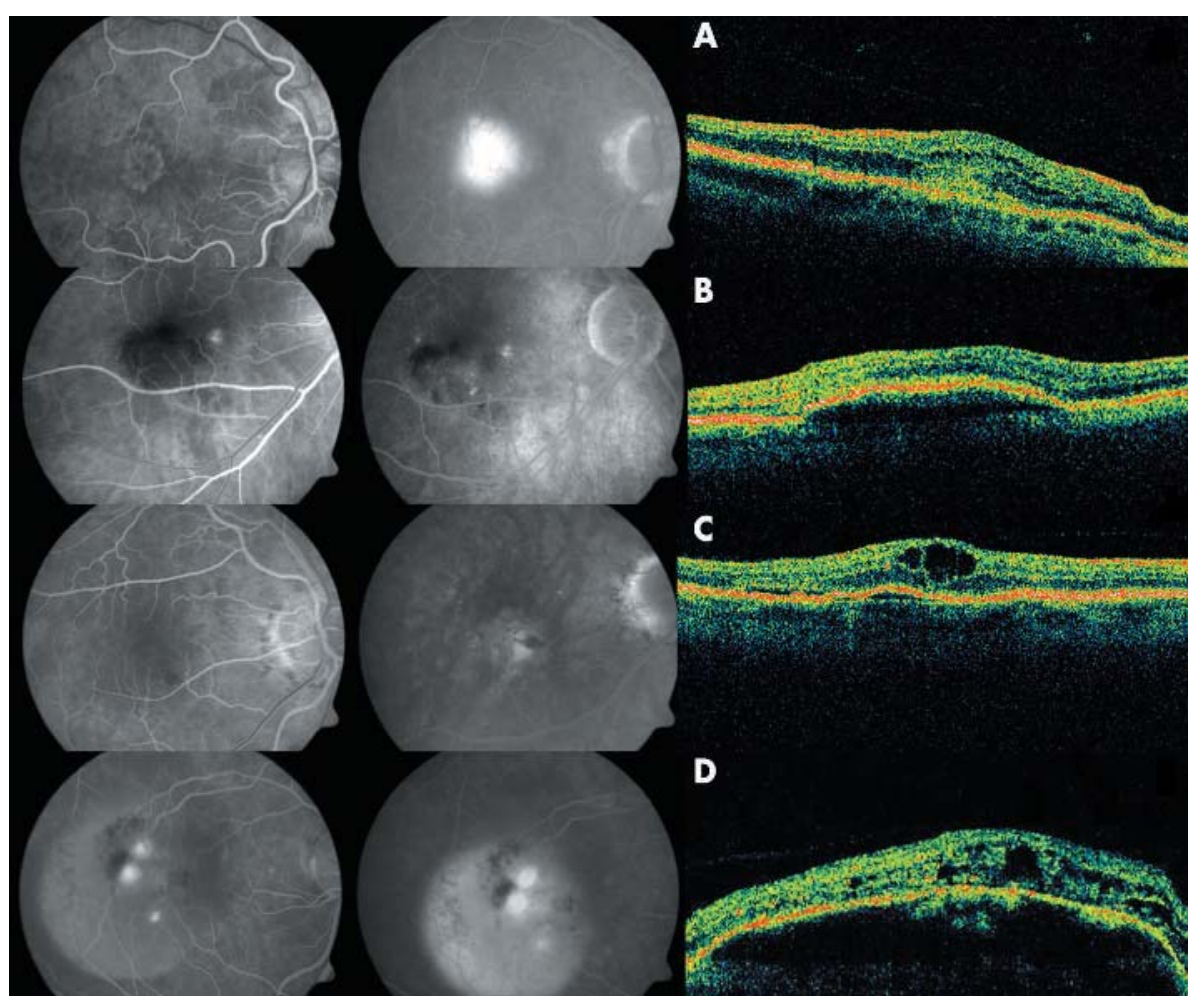

Figure 1 The typical FFA and OCT appearances of classic CNV, occult CNV, and serous PED. (A) Illustration of classic CNV: FFA and OCT. (B) Illustration of occult CNV, vascularised PED: FFA and OCT. (C) Illustration of occult CNV, late leak: FFA and OCT. (D) Illustration of serous PED with two small classic spots: FFA and OCT. 
drusen/atrophy FFA diagnoses, which on further review of the OCT, still appeared to be highly characteristic of new CNV with SRF. These findings highlight that OCT findings could suggest SRF but FFA findings show no active leak. We believe that these cases highlight a grey area when trying to differentiate between stain and active leak on FFA. While FFA remains the gold standard for active leak, it may be that the OCT is more sensitive and better at defining structure.

Defining components of $\mathrm{CNV}$ was complex. Late leak occult CNVs were hard to characterise, appearing to have components of classic CNV, cystoid, and PED (fig lC). Indeed, a fifth of occult CNVs were classified as classic CNV by OCT. It was difficult to distinguish a serous from a vascularised PED with the OCT, as the scan is unable to penetrate the RPE (fig 1B, 1D, table 2).

The range of patients analysed in this study may not represent the true proportion of types of CNV presenting to a hospital, as the group imaged represents that thought likely to have a potentially treatable form of CNV under present UK provision. ${ }^{15}$ However, a range of $\mathrm{CNV}$ lesion types were imaged, allowing diagnostic accuracy to be assessed.

Forty seven of 131 eyes (35.9\%) did not have FFA evidence of exudative AMD and 21 of $131(16.0 \%)$ had a non-AMD related diagnosis. This suggests that in a busy department with a range of doctors assessing the patients, there is a level of inaccurate initial diagnosis. However, CNV may lack clear symptoms and signs, and therefore requests for further investigation of potentially treatable lesions are often warranted.

Our results suggest that the OCT is very good at picking up potentially treatable AMD as only 3.6\% of the FFAs showing new AMD lesions were classified as non-CNV by OCT alone. However it is not so good at differentiating between classic and occult CNV lesions. OCT diagnostic accuracy, compared to FFA as gold standard, is improved with the use of additional colour photographs. However, it is not good enough to replace FFA. Our results suggest OCT could be used at least to prioritise further assessment of patients and so avoid delays in patients most likely to benefit from treatment.

\section{Authors' affiliations}

S S Sandhu, S J Talks, Department of Ophthalmology, Royal Victoria Infirmary, Newcastle upon Tyne, UK

Competing interests: none declared
Ethics committee approval not required.

Presented in part as a poster at the Association for Research in Vision and Ophthalmology meeting, Fort Lauderdale, FL, USA, May 2004.

Correspondence to: Mr S J Talks, Department of Ophthalmology, Royal Victoria Infirmary, Queen Victoria Road, Newcastle upon Tyne NE1 4LP, UK; james.talks@ncl.ac.uk

Accepted for publication 4 January 2005

\section{REFERENCES}

1 Hee MR, Baumal CR, Puliafito CA, et al. Optical coherence tomography of age-related macular degeneration and choroidal neovascularisation. Ophthalmology 1996;103:1260-70.

2 Rogers AH, Martidis A, Greenberg PB, et al. Optical coherence tomography findings following photodynamic therapy of choroidal neovascularisation. Am J Ophthalmol 2002;134:566-76.

3 Puliafito CA, Hee MR, Lin CP, et al. Imaging of macular diseases with optical coherence tomography. Ophthalmology 1995;102:217-29.

4 Hee MR, Puliafito CA, Wong C, et al. Optical coherence tomography of macular holes. Ophthalmology 1995;102:748-56.

5 Hee MR, Puliafito CA, Duker JS, et al. Topography of diabetic macular oedema with optical coherence tomography. Ophthalmology 1998; 105:360-70.

6 Schuman JS, Hee MR, Arya AV, et al. Optical coherence tomography: a new tool for glaucoma diagnosis. Curr Opin Ophthalmol 1995;6:89-95.

7 Macular Photocoagulation Study Group. Subfoveal neovascular lesions in age-related macular degeneration. Arch Ophthalmol 1991;109:1242-57.

8 Treatment of Age-related Macular Degeneration with Photodynamic Therapy (TAP) Study Group. Photodynamic therapy of subfoveal choroidal neovascularisation in age-related macular degeneration with verteporfin. One year results of 2 randomised clinical trials-TAP Report 1. Arch Ophthalmol 1999; 117:1329-45.

9 Treatment of Age-related Macular Degeneration with Photodynamic Therapy (TAP) Study Group. Photodynamic therapy of subfoveal choroidal neovascularisation in age-related macular degeneration with verteporfin. Two year results of 2 randomised clinical trials-TAP Report 2. Arch Ophthalmol 2001;119:198-207.

10 Verteporfin in Photodynamic Therapy (VIP) Study Group. Photodynamic therapy of subfoveal choroidal neovascularisation in pathological myopia with verteporfin: one year results of a randomised clinical trial-VIP Report Number 1. Ophthalmology 2001;108:841-52.

11 Verteporfin in Photodynamic Therapy (VIP) Study Group. Verteporfin therapy of subfoveal choroidal neovascularisation in age-related macular degeneration: two year results of a randomised clinical trial including lesions with occult with no classic choroidal neovascularisation-VIP Report Number 2. Am J Ophthalmol 2001;131:541-60.

12 Hee MR, Izait JA, Swanson EA, et al. Optical coherence tomography of the human retina. Arch Ophthalmol 1995;113:325-32.

13 Schuman JS, Puliafito CA, Fujimoto JG. Age-related macular degeneration. in optical coherence tomography of ocular diseases, 2nd ed. New York: Slack Incorporated, 2004:243-344.

14 Talks SJ, Setty R, Clarke C. The incidence and outcome of photodynamic therapy for macular degeneration in the northern region of the UK. Eye 2004; 18:588-94

15 Olse T, Feng $X$, Kasper $T$, et al. Fluorescein angiographic lesion type frequency in neovascular age-related macular degeneration. Ophthalmology $2004 ; 111: 250-5$ 